\title{
The Analysis of Crossed Foundation Beams Lying on the Soil Base
}

\author{
Teimuraz Kikava, Levan Dolidze \\ Engineering and Construction Department, Faculty of Technologies, Batumi Shota Rustaveli State University, Batumi, Georgia \\ Email address: \\ temurikikava@yahoo.com (T. Kikava)

\section{To cite this article:} \\ Teimuraz Kikava, Levan Dolidze. The Analysis of Crossed Foundation Beams Lying on the Soil Base. American Journal of Civil \\ Engineering. Vol. 9, No. 1, 2021, pp. 25-30. doi: 10.11648/j.ajce.20210901.14
}

Received: January 20, 2021; Accepted: February 14, 2021; Published: March 26, 2021

\begin{abstract}
To ensure uniform settlement of buildings and structures erected on a weak and heterogeneous soils, reinforced concrete foundations are designed from cross beams, or solid slabs. Currently, cross foundation beams and solid slabs are widely used in construction, like in the construction of high-rise buildings and many other engineering structures. In cross beams, at their intersection with an increase in load and stiffness, especially with an asymmetric load, in addition to bending moments and shear forces, the torques reaching large values can also occur, which are often not taken into account. The torques arisen at the knots of the crossed foundations may, in some cases, have a significant effect on their operation, for example, if the torques reach important values, then additional reinforcement must be placed in the foundations by stipulating the pressures arisen from them. For the correct selection of reinforcement, it is necessary to know the values of not only the bending moments, but also the torques arisen in the constructions. The existing methods of calculation often consider and calculate the crossed foundations as the constructions lying on a linearly deformable base. Besides most of them do not take into account the influence of torques. The methods of calculating the crossed foundations have been worked out by the author, by stipulating effect of bending moments on their working. There are given the formulas to calculate the bending moments, the system of equations is drawn up for linear and angular movements to define the unknown forces arisen at the knots of crossing the foundation beams. After determining these unknown forces, each beam is considered and calculated as a separate one, lying on a linearly deformable foundation.
\end{abstract}

Keywords: Crossed Foundations, Linearly Deformable Base, Torques, Bending Moments

\section{Introduction}

Today there is no unified theory for calculating the crossed foundations of the high buildings and the other engineering constructions. This leads to the fact that if it is necessary to design such structures, it is necessary to simplify the calculation schemes and therefore the results of calculations often turn out to be incorrect, therefore, the development of practical methods of calculating the crossed foundations has a great scientific and applied importance.

The torques arisen at the knots of the crossed foundations may, in some cases, have a significant effect on their operation, for example, if the torques reach important values, then additional reinforcement must be placed in the foundations by stipulating the pressures arisen from them. For the correct selection of reinforcement, it is necessary to know the values of not only the bending moments, but also the torques arisen in the constructions.

The existing methods of calculation often consider and calculate the crossed foundations as the constructions lying on a linearly deformable base. Besides most of them do not take into account the influence of torques.

Proceeding from the foregoing, the purpose of this work is to develop a practical methodology of calculating these types of foundations, taking into account the effect of torque on the operation of construction.

In most cases, the methods of calculating the crossed foundations neglect the torque generated at the intersection points of the bands, which can have a significant effect on the calculation results. The aim of this work is to develop such methodology for calculating cross-sectional foundations which could take this factor into account. When calculating, 
we use the expressions given in [1-15].

\section{The Exposition of Calculation Method}

Let's consider the elementary section $d x$ of a beam on a linearly deformable base. Using the formula (5-40) (Simvulidi, I. 1973) [2], we find the twist angle $\varphi_{X}^{K}$ of the element dx:

$$
\varphi_{X}^{K}=\bar{\varphi} \frac{d M_{X}^{K}}{\pi \cdot E_{0} \cdot d x \cdot b^{2}} \varsigma 6 \frac{d M_{X}^{K}}{d x}=\frac{\pi \cdot E_{0} \cdot b^{2}}{\bar{\varphi}} \varphi_{X}^{K}
$$

Where $\bar{\varphi}$ is the product of the flexibility index $\alpha$ on the abstract quantities included in the curly brackets of the formula (5-39) [2] (Simvulidi, I. 1973).

However, according to Saint-Venant [3], the elementary twist angle $d \varphi_{X}$ is determined by the well-known formula from the theory of torsion:

$$
d \varphi_{X}^{K}=\frac{M_{X}^{K} \cdot d x}{V \cdot G \cdot b \cdot h^{3}} \varsigma 5 \frac{d \varphi_{X}^{K}}{d x}=\frac{M_{X}^{K}}{V \cdot G \cdot b \cdot h^{3}}
$$

where $\mathrm{G}$ is the shear modulus.

$\mathrm{M}^{\mathrm{K}}$ denotes torques

$\mathrm{M}^{\mathrm{I}}$ - bending moments

The values of $\mathrm{V}$ for different relations among the sides of the rectangle are taken from the Table of B. Saint-Venant [3]:

\begin{tabular}{|c|c|c|c|c|c|c|c|c|c|c|c|}
\hline b/h & 1 & 1,5 & 1,75 & 2 & 2,5 & 3 & 4 & 6 & 8 & 10 & $\infty$ \\
\hline $\mathrm{V}$ & 0,208 & 0,231 & 0,239 & 0,246 & 0,258 & 0,267 & 0,282 & 0,299 & 0,307 & 0,312 & 0,333 \\
\hline
\end{tabular}

Table 1. V value for various ratios between the sides of the rectangle.

By taking the first derivative from $d \varphi_{X}^{K} / d x$ in $\mathrm{X}$ [2] and solving the received expression towards $d M_{X}^{K} / d x$, we obtain:

$$
\frac{d M_{X}^{K}}{d x}=V \cdot G \cdot b \cdot h^{3} \frac{d^{2} \varphi_{X}^{K}}{d x^{2}}
$$

By equating the right-hand sides of equations (1) - (3), we obtain:

$$
\begin{gathered}
\left.V \cdot G \cdot b \cdot h^{3} \frac{d^{2} \varphi_{X}^{K}}{d x^{2}}=\frac{\pi \cdot E_{0} \cdot b^{2}}{\bar{\varphi}} \varphi_{X}^{K}\right\lrcorner 5 \frac{d^{2} \varphi_{X}^{K}}{d x^{2}}-k^{2} \cdot \varphi_{X}^{K}=0 \\
\text { Where } k^{2}=\pi \cdot b \cdot E_{0} /\left(\bar{\varphi} \cdot V \cdot G \cdot h^{3}\right)
\end{gathered}
$$

Expression (4) is the differential equation of the torques of the beam on an elastic foundation (Ovechkin, O. 1936) [5]

By solving equation (3) with respect to $\varphi_{X}^{K}$, we obtain:

$$
\varphi_{X}^{K}=A \operatorname{sh}(k x)+B \operatorname{ch}(k x)
$$

The first derivative of this expression has the form:

$$
\frac{d \varphi_{X}^{K}}{d x}=k[A \operatorname{ch}(k x)+B \operatorname{sh}(k x)]
$$

By substituting the value $d \varphi_{X}^{K} / d x$ in equation (2), we obtain:

$$
\frac{M_{X}^{K}}{V \cdot G \cdot b \cdot h^{3}}=k[A \operatorname{ch}(k x)+B \operatorname{sh}(k x)] \text { or }
$$

To determine the integration constants $\mathrm{A}$ and $\mathrm{B}$, we use the following conditions: When $X=0 M_{K}=M_{0}^{K}$ and $\varphi=\varphi_{0}$ By using these conditions and (6) and (8) equations, we find $\varphi_{0}^{K}=B$ and $M_{0}^{K}=k \cdot V \cdot G \cdot b \cdot h^{3}=A$ By substituting the found values of $\mathrm{A}$ and $\mathrm{B}$ into equations (6) and (8), we obtain:

$$
\begin{gathered}
\varphi_{X}^{K}=\varphi_{0}^{K} \cdot \operatorname{ch}(k x)+\rho M_{0}^{K} \operatorname{sh}(k x) \\
M_{X}^{K}=M_{0}^{K} \cdot \operatorname{ch}(k x)+\left(\varphi_{0}^{K} / \rho\right) \cdot \operatorname{sh}(k x)
\end{gathered}
$$

Where coefficient

$$
\rho=\frac{1}{k \cdot V \cdot G \cdot b \cdot h^{3}}
$$

Let's consider the cross beams lying on the linearly deformable base (drawing 1). Let's suppose that all the nodes $11,21,31, \ldots \mathrm{mn}$, forming the cross-beams, are rigid, then in each node there will be six unknown forces. To determine these unknown forces, for each node we compose three equilibrium equations of statics and three compatibility equations for linear and angular displacements.

The equations of linear displacements in the nodes with the coordinates $11,21, \ldots, \mathrm{m} 1, \ldots, 1 \mathrm{n}, 2 \mathrm{n}, \ldots, \mathrm{mn} \mathrm{A} 1 \mathrm{~B} 1$, $\mathrm{A} 2 \mathrm{~B} 2, \ldots, \mathrm{AnBn}$, for the beams have the form:

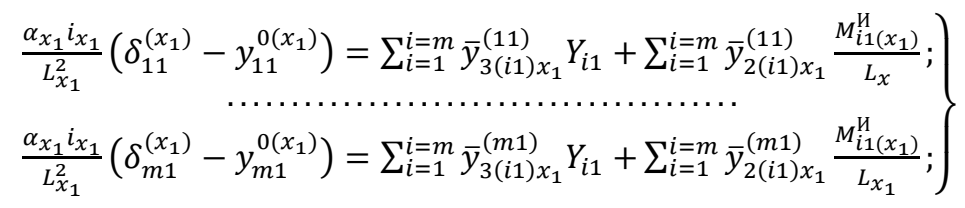

$$
\begin{aligned}
& \left.\begin{array}{c}
\frac{\alpha_{x_{n}} i_{x_{n}}}{L_{x_{n}}^{2}}\left(\delta_{1 n}^{\left(x_{n}\right)}-y_{1 n}^{0\left(x_{n}\right)}\right)=\sum_{i=1}^{i=m} \bar{y}_{3(i n) x_{n}}^{(1 n)} Y_{i n}+\sum_{i=1}^{i=m} \bar{y}_{2(i n) x_{n}}^{(1 n)} \frac{M_{i n\left(x_{n}\right)}^{U}}{L_{x_{n}}} ; \\
\cdots \cdots \cdots \cdots \cdots \cdots \cdots \cdots \cdots \cdots \cdots \cdots \cdots \cdots \cdots \cdots \cdots \cdots \cdots \cdots \cdots \cdots \cdots \cdots \\
\frac{\alpha_{x_{n}} i_{x_{n}}}{L_{x_{n}}^{2}}\left(\delta_{m n}^{\left(x_{n}\right)}-y_{m n}^{0\left(x_{n}\right)}\right)=\sum_{i=1}^{i=m} \bar{y}_{3(i n) x_{n}}^{(m n)} Y_{i n}+\sum_{i=1}^{i=m} \bar{y}_{2(i n) x_{n}}^{(m n)} \frac{M_{i n\left(x_{n}\right)}^{h}}{L_{x_{n}}} ;
\end{array}\right\}
\end{aligned}
$$

Nodal forces N11, N21,... Nmn cause unknown forces Y11, Y21,..., Ymn on the beams parallel to the axis OX and unknown forces $Y_{11}^{\prime}, Y_{21}^{\prime}, \ldots, Y_{m n}^{\prime}$ on the beams parallel to the $\mathrm{OZ}$ axis. The relationship between these forces can be expressed by the 
formula:

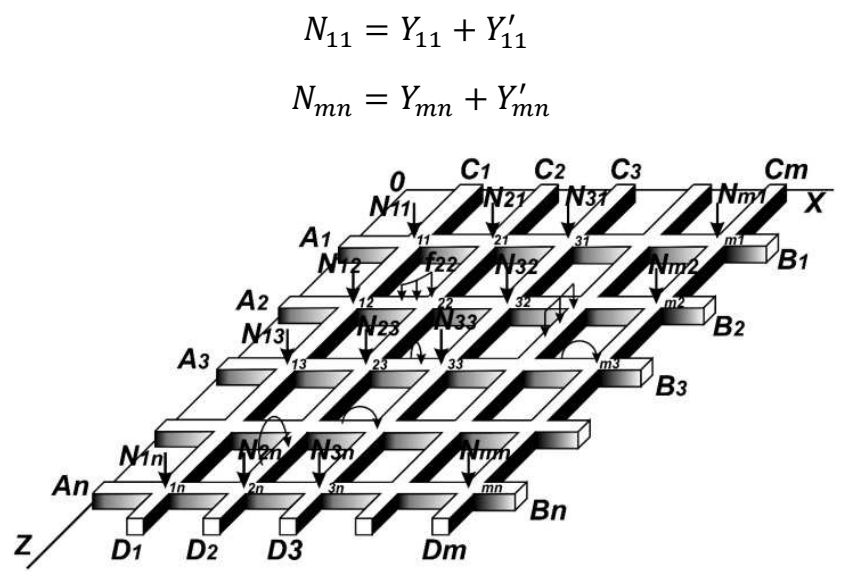

Figure 1. Calculation scheme of the crossed foundation beams.

If the indices $\mathrm{Xn}$ are replaced everywhere by the indices $\mathrm{Zm}$ in the equations (12) and (13), and also $Y_{11}^{\prime}, Y_{21}^{\prime}, \ldots, Y_{m n}^{\prime}$ are replaced by N11-Y11, N21-Y21, we obtain equations for beams parallel to the $0 \mathrm{Z}$ axis $(\mathrm{C} 1 \mathrm{D} 1, \ldots, \mathrm{CmDm})$. Therefore, it is not necessary to give the equations of linear displacements for beams parallel to the $0 \mathrm{Z}$ axis. By stipulating the equations of compatibility of linear displacements, we have:

$$
\left.\begin{array}{c}
\lambda_{11}\left[\sum_{i=1}^{i=m} \bar{y}_{3(i 1) x_{1}}^{(11)} Y_{i 1}+\sum_{i=1}^{i=m} \bar{y}_{2(i 1) x_{1}}^{(11)} \frac{M_{i 1(x)}^{h}}{L_{x_{1}}}\right]+\sum_{j=1}^{j=m} \bar{y}_{3(1 j) z_{1}}^{(11)}\left(Y_{1 j}-N_{1 j}\right)+\sum_{j=1}^{j=n} \bar{y}_{2(i j) z_{1}}^{(11)} \frac{M_{1 j(z)}^{\mathrm{U}}}{L_{z_{1}}}==\frac{\alpha_{z_{1}} i_{z_{1}}}{L_{z_{1}}^{2}}\left(\bar{y}_{11}^{0\left(z_{1}\right)}-\bar{y}_{11}^{0\left(x_{1}\right)}\right) ; \\
\lambda_{m 1}\left[\sum_{i=1}^{i=m} \bar{y}_{3(i 1) x_{1}}^{(m 1)} Y_{i 1}+\sum_{i=1}^{i=m} \bar{y}_{2(i 1) x_{1}}^{(m 1)} \frac{M_{i 1(x)}^{h}}{L_{x_{1}}}\right]+\sum_{j=1}^{j=m} \bar{y}_{3(m j) z_{m}}^{(m 1)}\left(Y_{m j}-N_{m j}\right)+\sum_{j=1}^{j=n} \bar{y}_{2(m j) z_{m}}^{(m 1)} \frac{M_{m j(z)}^{h}}{L_{z_{m}}}==\frac{\alpha_{z_{m}} i_{z_{m}}}{L_{z_{m}}^{2}}\left(\bar{y}_{m 1}^{0\left(z_{m}\right)}-\bar{y}_{m 1}^{0\left(x_{1}\right)}\right) ;
\end{array}\right\}
$$

In these equations $\alpha$ is the index of flexibility:

$$
\alpha=\frac{1-\mu^{2}}{1-\mu_{0}^{2}} \cdot 12 \pi \frac{E_{0}}{E}\left(\frac{L}{h}\right)^{3}
$$

$\mathrm{E}_{0}$ - modulus of deformation of soil;

E - modulus of elasticity of the structure;

$\mu$ - the Poisson ratio;

Coefficient:

$$
\lambda_{m n}=\frac{\alpha_{Z_{m}}}{\alpha_{X_{n}}} \cdot \frac{i_{Z_{m}}}{i_{X_{n}}} \cdot \frac{L_{X_{n}}^{2}}{L_{Z_{m}}^{2}}=\frac{b_{Z_{m}}}{b_{X_{n}}} \cdot \frac{E_{0 Z_{m}}}{E_{0 X_{n}}}
$$

$\mathrm{b}$ and $\mathrm{L}$, respectively, are the width and length of the beam.

$i=\frac{E I}{L}$ is the linear stiffness of the structure.

$$
\begin{gathered}
y_{m n}^{0\left(X_{n}\right)}=\frac{L_{X_{n}}}{\alpha_{X_{n}} \cdot i_{X_{n}}} \cdot\left(b_{X_{n}} \cdot L_{X_{n}}^{2} \cdot \sum \bar{y}_{H_{i}\left(X_{n}\right)}^{(m n)} \cdot q_{i}+\sum y_{2 i\left(X_{n}\right)}^{(m n)} \cdot M_{i}+L_{X_{n}} \cdot \sum \bar{y}_{3_{i}\left(X_{n}\right)}^{(m n)} \cdot P_{i}\right) \\
y_{m n}^{0\left(Z_{m}\right)}=\frac{L_{Z_{m}}}{\alpha_{Z_{m}} \cdot i_{Z_{m}}} \cdot\left(b_{Z_{m}} \cdot L_{Z_{m}}^{2} \cdot \sum \bar{y}_{H_{i}\left(Z_{m}\right)}^{(m n)} \cdot q_{i}+\sum y_{2 i\left(Z_{m}\right)}^{(m n)} \cdot M_{i}+L_{Z_{m}} \cdot \sum \bar{y}_{3_{i}\left(Z_{m}\right)}^{(m n)} \cdot P_{i}\right)
\end{gathered}
$$

$\bar{y}_{3(i n)}^{(m n)}$ - deflections caused in $m n$ node from the action of Ymn force at in node, which are taken from the tables [2], according to the flexibility index $\alpha$, the place of application of the load $\beta$ and the section $\xi$ under consideration.

$\delta_{m n}^{\left(X_{n}\right)}-$ is the linear displacement of $A_{n} B_{n}$ beam at $X_{n}$ node.

$\bar{y}_{2(i n)}^{(m n)}$ - deflections in $m n$ node caused by action of the bending moment $M$ at the node in.

Equations for the beams intersecting at the nodes with coordinates $1 \mathrm{n}, 2 \mathrm{n}, \ldots, \mathrm{mn}$, have the following form: 


$$
\left.\begin{array}{c}
\lambda_{1 n}\left[\sum_{i=1}^{i=m} \bar{y}_{3(i n) x_{n}}^{(1 n)} Y_{i n}+\sum_{i=1}^{i=m} \bar{y}_{2(i n) x_{n}}^{(1 n)} \frac{\left.M_{i n(x)}^{\mathrm{h}}\right]}{L_{x_{n}}}\right]+\sum_{j=1}^{j=n} \bar{y}_{3(1 j) z_{1}}^{(1 n)}\left(Y_{1 j}-N_{1 j}\right)+\sum_{j=1}^{j=n} \bar{y}_{2(1 j) z_{1}}^{(1 n)} \frac{M_{1 j(\mathrm{z})}^{\mathrm{U}}}{L_{z_{1}}}=\frac{\alpha_{z_{1}} i_{z_{1}}}{L_{z_{1}}^{2}}\left(y_{1 n}^{0\left(z_{1}\right)}-y_{1 n}^{0\left(x_{n}\right)}\right) ; \\
\lambda_{m n}\left[\sum_{i=1}^{i=m} \bar{y}_{3(i n) x_{n}}^{(m n)} Y_{i 1}+\sum_{i=1}^{i=m} \bar{y}_{2(i n) x_{n}}^{(m n)} \frac{\left.M_{i n(x)}^{\mathrm{U}}\right]}{L_{x_{n}}}\right]++\sum_{j=1}^{j=n} \bar{y}_{3(m j) z_{m}}^{(m n)}\left(Y_{m j}-N_{m j}\right)+\sum_{j=1}^{j=n} \bar{y}_{2(m j) z_{m}}^{(m n)} \frac{M_{m j(z)}^{\mathrm{U}}}{L_{z_{m}}}=\frac{\alpha_{z_{m}} i_{z_{m}}}{L_{z_{m}}^{2}}\left(y_{m n}^{0\left(z_{m}\right)}-y_{m n}^{0\left(x_{n}\right)}\right) ;
\end{array}\right\}
$$

Let's formulate the compatibility equations for angular displacements for the cases when crossbeams parallel to the $\mathrm{X}$ axis under the action of forces bend and the beams parallel to the $\mathrm{Z}$ axis, in connection with the presence of rigid bonds at the nodes, are twisted. Equations of consistency of angular displacements for the beams intersecting in the nodes with coordinates $11,21, \ldots, \mathrm{m} 1$, have the following form:

$$
\left.\begin{array}{c}
\frac{L_{x_{1}}}{\alpha_{x_{1}} i_{x_{1}}}\left[\sum_{i=1}^{i=m} \bar{\varphi}_{3(i 1) x_{1}}^{(11)} Y_{i 1}+\frac{1}{L_{x_{1}}} \sum_{i=1}^{i=m} \bar{\varphi}_{2(i 1) x_{1}}^{(11)} M_{i 1\left(x_{1}\right)}^{\mathrm{U}}\right]+\varphi_{11}^{0\left(x_{1}\right)}=\rho_{11\left(z_{1}\right)} \frac{\operatorname{chk_{11}(z_{1})} l_{11\left(z_{1}\right)}}{s h k_{11\left(z_{1}\right)} l_{11\left(z_{1}\right)}} M_{11\left(z_{1}\right)}^{K} \\
\frac{L_{x_{1}}}{a_{x_{1}} i_{x_{1}}}\left[\sum_{i=1}^{i=m} \bar{\varphi}_{3(i 1) x_{1}}^{(m 1)} Y_{i 1}+\frac{1}{L_{x_{1}}} \sum_{i=1}^{i=m} \bar{\varphi}_{2(i 1) x_{1}}^{(m 1)} M_{i 1\left(x_{1}\right)}^{U}\right]++\varphi_{m 1}^{0\left(x_{1}\right)}=\rho_{m 1\left(z_{m}\right)} \frac{c h k_{m 1\left(z_{m}\right)} l_{m 1\left(z_{m}\right)}}{s h k_{m 1\left(z_{m}\right)} l_{m 1}\left(z_{m}\right)} M_{m 1\left(z_{m}\right)}^{K}
\end{array}\right\}
$$

Equations of compatibility of angular displacements for the beams intersecting at the nodes with coordinates $1 \mathrm{n}, 2 \mathrm{n}, \ldots, \mathrm{mn}$, have the following form:

$$
\begin{aligned}
& \frac{L_{x_{n}}}{\alpha_{x_{n}} i_{x_{n}}}\left[\sum_{i=1}^{i=m} \bar{\varphi}_{3(i n) x_{n}}^{(1 n)} Y_{i n}+\frac{1}{L_{x_{n}}} \sum_{i=1}^{i=m} \bar{\varphi}_{2(i n) x_{n}}^{(1 n)} M_{i n\left(x_{n}\right)}^{U}\right]+ \\
& +\varphi_{1 n}^{0\left(x_{n}\right)}=\rho_{1 n\left(z_{1}\right)}\left[\frac{\operatorname{ch} k_{1 n\left(z_{1}\right)} l_{11\left(z_{1}\right)}}{\operatorname{sh} k_{1 n\left(z_{1}\right)} l_{11\left(z_{1}\right)}} M_{11\left(z_{1}\right)}^{K}++\frac{\operatorname{sh} k_{1 n\left(z_{1}\right)}\left(l_{12\left(z_{1}\right)}-l_{11\left(z_{1}\right)}\right)}{1+\operatorname{ch} k_{1 n\left(z_{1}\right)}\left(l_{12\left(z_{1}\right)}-l_{11\left(z_{1}\right)}\right)} M_{12\left(z_{1}\right)}^{K}+\ldots+\frac{\operatorname{sh} k_{1 n\left(z_{1}\right)}\left(l_{1 n\left(z_{1}\right)}-l_{1(n-1)\left(z_{1}\right)}\right)}{1+c h k_{1 n\left(z_{1}\right)}\left(l_{1 n\left(z_{1}\right)}-l_{1(n-1)\left(z_{1}\right)}\right)} M_{1 n\left(z_{1}\right)}^{K}\right] ; \\
& \frac{L_{x_{n}}}{\alpha_{x_{n}} i_{x_{n}}}\left[\sum_{i=1}^{i=m} \bar{\varphi}_{3(i n) x_{n}}^{(m n)} Y_{m n}+\frac{1}{L_{x_{n}}} \sum_{i=1}^{i=m} \bar{\varphi}_{2(i n) x_{n}}^{(m n)} M_{i n\left(x_{n}\right)}^{\mathrm{U}}\right]+
\end{aligned}
$$

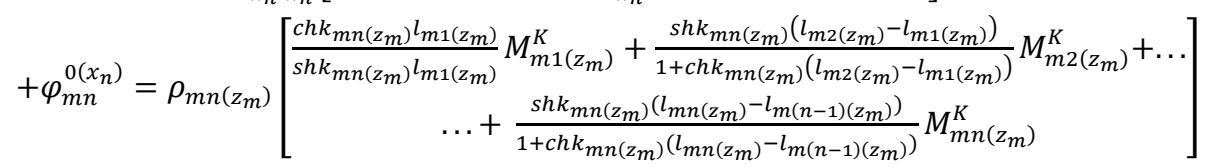

Let's compose the equations of consistency of angular displacements for the cases when the crossbeams parallel to the $\mathrm{Z}$ axis under the action of forces bend and the beams parallel to the $\mathrm{X}$ axis, in connection with the presence of rigid bonds at all the nodes, are twisted.

Equations of consistency of angular displacements for the beams intersecting at the nodes with coordinates $11,12, \ldots, 1 \mathrm{n}$, have the following form:

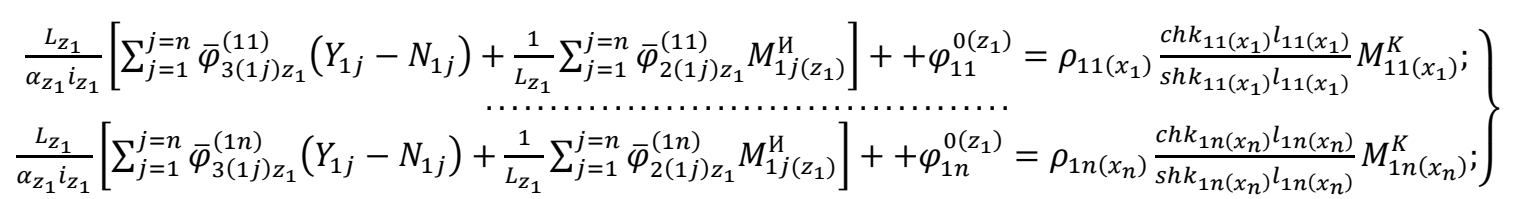

Equations of consistency of angular displacements for the beams intersecting at the nodes with coordinates $\mathrm{m} 1, \mathrm{~m}^{2}, \ldots, \mathrm{mn}$, have the following form:

$$
\begin{aligned}
& \frac{L_{z_{m}}}{\alpha_{z_{m}} i_{z_{m}}}\left[\sum_{j=1}^{j=n} \bar{\varphi}_{3(m j) z_{m}}^{(m 1)}\left(Y_{m j}-N_{m j}\right)+\frac{1}{L_{z_{m}}} \sum_{i=1}^{j=n} \bar{\varphi}_{2(m j) z_{m}}^{(m 1)} M_{m j\left(z_{m}\right)}^{U}\right]+ \\
& \varphi_{m 1}^{0\left(z_{m}\right)}=\rho_{m 1\left(x_{1}\right)}\left[\frac{\operatorname{ch} k_{m 1\left(x_{1}\right)} l_{11\left(x_{1}\right)}}{\operatorname{sh} k_{m 1\left(x_{1}\right)} l_{11\left(x_{1}\right)}} M_{11\left(x_{1}\right)}^{K}++\frac{\operatorname{sh} k_{m 1\left(x_{1}\right)}\left(l_{21\left(x_{1}\right)}-l_{11\left(x_{1}\right)}\right)}{1+\operatorname{ch} k_{m 1}\left(x_{1}\right)\left(l_{21\left(x_{1}\right)}-l_{11\left(x_{1}\right)}\right)} M_{21\left(x_{1}\right)}^{K}+\ldots+\frac{\operatorname{sh} k_{m 1\left(x_{1}\right)}\left(l_{m 1\left(x_{1}\right)}-l_{(m-1) 1\left(x_{1}\right)}\right)}{1+\operatorname{ch} k_{m 1\left(x_{1}\right)}\left(l_{m 1\left(x_{1}\right)}-l_{(m-1) 1\left(x_{1}\right)}\right)} M_{m 1\left(x_{1}\right)}^{K}\right]
\end{aligned}
$$

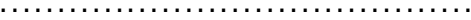

$$
\begin{aligned}
& \left.\left[\begin{array}{c}
\frac{\operatorname{ch} k_{m n\left(x_{n}\right)} l_{1 n\left(x_{n}\right)}}{\operatorname{sh} k_{m n n}\left(x_{n}\right) l_{1 n\left(x_{n}\right)}} M_{1 n\left(x_{n}\right)}^{K}+ \\
\frac{\operatorname{sh} k_{m n\left(x_{n}\right)}\left(l_{2 n\left(x_{n}\right)}-l_{1 n\left(x_{n}\right)}\right)}{1+\operatorname{ch} k_{m n\left(x_{n}\right)}\left(l_{2 n\left(x_{n}\right)}-l_{1 n\left(x_{n}\right)}\right)} M_{2 n\left(x_{n}\right)}^{K}+. . \\
+\frac{\operatorname{sh} k_{m n\left(x_{n}\right)}\left(l_{m n\left(x_{n}\right)}-l_{(m-1) n\left(x_{n}\right)}\right)}{1+\operatorname{chk} k_{m n\left(x_{n}\right)}\left(l_{m n\left(x_{n}\right)}-l_{(m-1) n\left(x_{n}\right)}\right)} M_{m n\left(x_{n}\right)}^{K}
\end{array}\right]\right\}
\end{aligned}
$$

If we change in (21)-(24) equations:

$$
\begin{aligned}
& M_{11\left(z_{1}\right)}^{K}=M_{11\left(x_{1}\right)}^{\mathrm{U}} ; M_{21\left(z_{2}\right)}^{K}=M_{21\left(x_{1}\right)}^{\mathrm{U}} ; \ldots ; M_{m 1\left(z_{m}\right)}^{K}=M_{m 1\left(x_{1}\right)}^{\mathrm{U}} ; \\
& M_{22\left(z_{2}\right)}^{K}=M_{22\left(x_{2}\right)}^{\mathrm{U}} ; M_{m 2\left(z_{m}\right)}^{K}=M_{m 2\left(x_{2}\right)}^{\mathrm{U}} ; \ldots ; M_{m n\left(z_{1}\right)}^{K}=M_{m n\left(x_{n}\right)}^{\mathrm{U}}
\end{aligned}
$$

and solve the obtained equations together with the equations (15) and (20), we find the values of $Y_{11}, Y_{21}, \ldots, Y_{m n}$;
$M_{11}^{K}, M_{21}^{K}, \ldots, M_{m n}^{K}$

After determining the bending moments that arise at the intersection points of the beams, we find the corresponding torques from them. By knowing the given loads-transverse forces, bending and twisting moments, each beam shall be calculated independently as a separate beam lying on a linearly deformable base [1]. After this, the diagrams of 
bending moments, soil reaction, transverse forces and deflections for each individual beam and then the diagram for the crossed foundation beams in general are constructed [4].

\section{Example of the Calculation Beam Lying on a Linearly Deformable Base}

Let us consider concrete example of the beam calculation (lower cross bar) on the linearly-deformed base loaded with the concentrated forces (Figure 2)

It is required to determinate values of curving moments $M$ and reaction pressures the ground $\rho$, if: $\mathrm{P} 1=\mathrm{P} 2=\mathrm{P} 3=\mathrm{P} 4=100 \mathrm{kN} ; \mathrm{PF}=200 \mathrm{kN} ; \mathrm{MA}=14 \mathrm{kNm}$; the width $\mathrm{b}=2 \mathrm{~m}$; the index of flexibility $\alpha=200$ the module of deformation $\mathrm{E} 0=320 \cdot 102 \mathrm{kN} / \mathrm{m}^{2}$

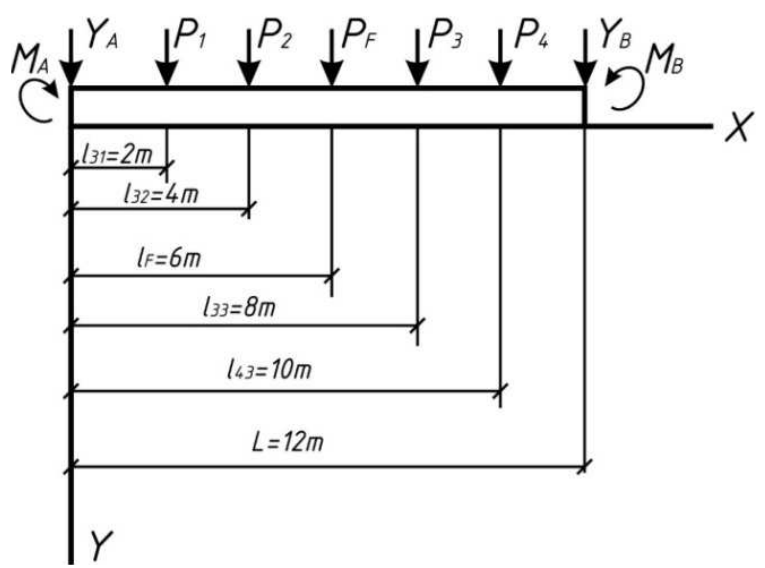

Figure 2. Calculation scheme of beam lying on a linearly deformable base.
Solution under the condition of the problem:

$$
\begin{gathered}
\beta_{1}=\frac{l_{31}}{L}=0,17 ; \\
\beta_{2}=\frac{l_{32}}{L}=0,33 ; \\
\beta_{F}=\frac{l_{F_{3}}}{L}=0,5 ; \\
\beta_{3}=\frac{l_{33}}{L}=0,67 ; \\
\beta_{4}=\frac{l_{43}}{L}=0,83 ;
\end{gathered}
$$

According to conditions of symmetry $\left|\mathrm{M}_{\mathrm{A}}\right|=\left|\mathrm{M}_{\mathrm{B}}\right|$ According to conditions of statics: $\mathrm{Y}_{\mathrm{A}}=\mathrm{Y}_{\mathrm{B}}=0$

For the determination of ordinate $\rho_{1} ; \rho_{2} ; \rho_{\mathrm{F}} ; \rho_{3} ; \rho_{4} ;$ from force $\mathrm{P}_{1} ; \mathrm{P}_{2} ; \mathrm{P}_{\mathrm{F}} ; \mathrm{P}_{3}$ and $\mathrm{P}_{4}$; we use the values of $\bar{\rho}_{1} ; \bar{\rho}_{2} ; \bar{\rho}_{1}$; $\bar{\rho}_{\mathrm{F}} ; \bar{\rho}_{3} ; \bar{\rho}_{4} ;$ corresponding $\alpha=200$ and $\beta_{1}=0,17 ; \beta_{2}=0,33$; $\beta_{\mathrm{F}}=0,5 ; \beta_{13}=0,67 ; \beta_{4}=0,83$; for all values $\bar{\rho}_{1} ; \bar{\rho}_{2} ; \bar{\rho}_{1}$; $\bar{\rho}_{\mathrm{F}} ; \bar{\rho}_{3} ;$ and $\bar{\rho}_{4} ;$ from $\xi=0$ till $\xi=1$, where $\xi$ is considered cross-section (Simvulidi, I.1987). Having multiplied each ordinate according to the formula $\rho=\bar{\rho} \cdot \mathrm{P} / \mathrm{b} \cdot L(13) ; \quad \mathrm{P} / \mathrm{bL}=100 / 2 \cdot 12=4,2 \mathrm{kN} / \mathrm{m}^{2}$

$\mathrm{P}_{\mathrm{F}} / \mathrm{bL}=200 / 24=8,4 \mathrm{kN} / \mathrm{m}^{2}$ we'll get values $\rho$ in various points.

\begin{tabular}{|c|c|c|c|c|c|c|c|c|c|c|c|}
\hline$\xi$ & 0 & 0,1 & 0,2 & 0,3 & 0,4 & 0,5 & 0,6 & 0,7 & 0,8 & 0,9 & 1,0 \\
\hline $\bar{\rho}_{1}$ & 3,845 & 2,793 & 1,988 & 1,388 & 0,956 & 0,648 & 0,424 & 0,246 & 0,068 & $-0,145$ & $-0,437$ \\
\hline$\rho_{1}$ & 16,149 & 11,731 & 8,35 & 5,83 & 4,02 & 2,72 & 1,781 & 1,033 & 0,29 & $-0,61$ & $-1,84$ \\
\hline $\bar{\rho}_{2}$ & 2,386 & 2,05 & 1,736 & 1,434 & 1,157 & 0,901 & 0,669 & 0,464 & 0,28 & 0,132 & 0,01 \\
\hline $\bar{\rho}_{F}$ & 0,666 & 0,846 & 0,987 & 1,087 & 1,147 & 1,167 & 1,147 & 1,087 & 0,987 & 0,846 & 0,666 \\
\hline$\rho_{\mathrm{F}}$ & 5,594 & 7,106 & 8,29 & 9,13 & 9,64 & 9,802 & 9,64 & 9,13 & 8,29 & 7,106 & 5,594 \\
\hline $\bar{\rho}_{3}$ & 0,01 & 0,132 & 0,28 & 0,464 & 0,669 & 0,901 & 1,157 & 1,434 & 1,736 & 2,05 & 2,386 \\
\hline$\rho_{3}$ & 0,04 & 0,55 & 1,18 & 1,95 & 2,81 & 3,784 & 4,86 & 6,023 & 7,29 & 8,61 & 10,02 \\
\hline $\bar{\rho}_{4}$ & $-0,437$ & $-0,145$ & 0,068 & 0,246 & 0,424 & 0,648 & 0,956 & 1,388 & 1,988 & 2,793 & 3,845 \\
\hline$\rho_{4}$ & $-1,84$ & $-0,61$ & 0,29 & 1,033 & 1,781 & 2,723 & 4,02 & 5,83 & 8,35 & 11,731 & 16,149 \\
\hline $\bar{\rho}_{M_{A}}$ & 15,323 & 7,758 & 2,572 & $-0,655$ & $-2,343$ & $-2,911$ & $-2,781$ & $-2,373$ & $-2,106$ & $-2,402$ & $-3,679$ \\
\hline $\bar{\rho}_{M_{B}}$ & $-3,679$ & $-2,402$ & $-2,106$ & $-2,373$ & $-2,781$ & $-2,911$ & $-2,343$ & $-0,655$ & $-2,572$ & $-7,758$ & $-15,323$ \\
\hline $\bar{\rho}_{M_{A}}+\bar{\rho}_{M_{B}}$ & 11,644 & 5,356 & 0,466 & $-3,028$ & $-5,124$ & $-5,822$ & $-5,124$ & $-3,028$ & 0,466 & 5,356 & 11,644 \\
\hline$\rho_{M_{A}}+\rho_{M_{B}}$ & 0,582 & 0,268 & 0,023 & $-0,151$ & $-0,256$ & $-0,291$ & $-0,256$ & $-0,151$ & 0,023 & 0,268 & 0,582 \\
\hline$\rho$ & 30,545 & 27,655 & 25,423 & 23,815 & 22,355 & 22,519 & 22,355 & 23,815 & 25,423 & 27,655 & 30,545 \\
\hline
\end{tabular}

Then we'll get values $\bar{\rho}_{M_{A}}$ and $\bar{\rho}_{M_{B}}$ for $\alpha=200 ; \beta_{21}=0$ and $\beta_{22}=1,0$ multiplied each ordinate to $\mathrm{M}_{\mathrm{A}} / \mathrm{bL}^{2}=14 / 2 \cdot 12^{2}$ $=0,05 \mathrm{kN} / \mathrm{m}^{2}$ and $\mathrm{M}_{\mathrm{B}} / \mathrm{bL}^{2}=0,05 \mathrm{kN} / \mathrm{m}^{2}$

Table 2. Values of soil reactions.

The values $\rho$ give in $\mathrm{kN} / \mathrm{m}^{2} ; \mathrm{M}-\mathrm{kN} \cdot \mathrm{m}$. Using the independence principle of force activity for getting values $\rho$ we take algebraic sum: $\rho=\rho_{1}+\rho_{2+} \rho_{\mathrm{F}+} \rho_{3+} \rho_{4+} \rho_{\mathrm{MA}+} \rho_{\mathrm{MB}}$

For the determination of ordinates curving moment $M$ from the forces $\mathrm{P}_{1} ; \mathrm{P}_{2} ; \mathrm{P}_{\mathrm{F}} ; \mathrm{P}_{3} ; \mathrm{P}_{4}$; and from moments $\mathrm{M}_{\mathrm{A}}$ and $\mathrm{M}_{\mathrm{B}}$, we use the values of $\bar{M}_{1} ; \bar{M}_{2} ; \bar{M}_{F} ; \bar{M}_{3} ; \bar{M}_{4} ; \bar{M}_{M_{A}}$ and $\bar{M}_{M_{B}}$ for $\alpha=200$ and $\beta_{1}=0,17 ; \beta_{2}=0,33 ; \beta_{\mathrm{F}}=0,5$; $\beta_{3}=0,67 ; \beta_{4}=0,83 ; \beta_{M_{A}}=0$ and $\beta_{M_{B}}=1,0$ for all values $\bar{M}$ from $\xi=0$ till $\xi=1$ (Simvulidi, I.1987). Having multiplied each ordinate to

$$
\left.\begin{array}{rl}
M_{P} & =\bar{M}_{P} \cdot P \cdot L \\
M_{M_{A}} & =\bar{M}_{M_{A}} \cdot M_{A} \\
M_{M_{B}} & =\bar{M}_{M_{B}} \cdot M_{B}
\end{array}\right\}
$$

$\mathrm{P} \cdot \mathrm{L}=100 \cdot 12=1200 \mathrm{kN} \cdot \mathrm{m} ; \mathrm{P}_{\mathrm{F}} \cdot \mathrm{L}=200 \cdot 112=2400 \mathrm{kN} \cdot \mathrm{m}$

Using the independence principle of force activity for getting values $\mathrm{M}$ we take algebraic sum:

$$
M=M_{P_{1}}+M_{P_{2}}+M_{P_{F}}+M_{P_{3}}+M_{P_{4}}+M_{A}+M_{B}
$$


Table 3. Values of bending moments.

\begin{tabular}{|c|c|c|c|c|c|c|c|c|c|c|c|}
\hline$\xi$ & 0 & 0,1 & 0,2 & 0,3 & 0,4 & 0,5 & 0,6 & 0,7 & 0,8 & $\mathbf{0 , 9}$ & 1,0 \\
\hline $\bar{M}_{P_{1}}$ & 0 & 0,018 & 0,063 & 0,029 & 0,008 & $-0,003$ & $-0,007$ & $-0,007$ & $-0,005$ & $-0,001$ & 0 \\
\hline$M_{P_{1}}$ & 0 & 21,6 & 75,6 & 34,8 & 9,6 & $-3,6$ & $-8,4$ & $-8,4$ & -6 & $-1,2$ & 0 \\
\hline $\bar{M}_{P_{2}}$ & 0 & 0,012 & 0,044 & 0,093 & 0,057 & 0,032 & 0,016 & 0,007 & 0,003 & 0,001 & 0 \\
\hline$M_{P_{2}}$ & 0 & 14,4 & 52,8 & 111,6 & 68,4 & 38,4 & 19,2 & 8,4 & 3,6 & 1,2 & 0 \\
\hline $\bar{M}_{P_{F}}$ & 0 & 0,004 & 0,015 & 0,037 & 0,07 & 0,114 & 0,07 & 0,037 & 0,015 & 0,004 & 0 \\
\hline$M_{P_{F}}$ & 0 & 9,6 & 36 & 88,8 & 168 & 273,6 & 168 & 88,8 & 36 & 9,6 & 0 \\
\hline $\bar{M}_{P_{3}}$ & 0 & 0,001 & 0,003 & 0,007 & 0,016 & 0,032 & 0,057 & 0,093 & 0,044 & 0,012 & 0 \\
\hline$M_{P_{3}}$ & 0 & 1,2 & 3,6 & 8,4 & 19,2 & 38,4 & 68,4 & 111,6 & 52,8 & 14,4 & 0 \\
\hline $\bar{M}_{P_{4}}$ & 0 & $-0,001$ & $-0,005$ & $-0,007$ & $-0,007$ & $-0,003$ & 0,008 & 0,029 & 0,063 & 0,018 & 0 \\
\hline$M_{P_{4}}$ & 0 & 1,2 & -6 & $-8,4$ & $-8,4$ & 3,6 & 9,6 & 34,8 & 75,6 & 21,6 & 0 \\
\hline $\bar{M}_{A}$ & -1 & $-0,937$ & $-0,796$ & $-0,625$ & $-0,460$ & $-0,318$ & $-0,204$ & $-0,119$ & $-0,054$ & $-0,015$ & 0 \\
\hline$\overline{\bar{M}}_{B}$ & 0 & $-0,015$ & $-0,054$ & $-0,119$ & $-0,204$ & $-0,318$ & $-0,460$ & $-0,625$ & $-0,796$ & $-0,937$ & -1 \\
\hline $\bar{M}_{M_{A}}+\bar{M}_{M_{B}}$ & -1 & $-0,952$ & $-0,85$ & $-0,744$ & $-0,664$ & $-0,636$ & $-0,664$ & $-0,744$ & $-0,85$ & $-0,952$ & -1 \\
\hline$M_{M_{A}}+M_{M_{B}}$ & -14 & $-13,33$ & $-11,9$ & $-10,42$ & $-9,3$ & $-8,9$ & $-9,3$ & $-10,42$ & $-11,9$ & $-13,33$ & -14 \\
\hline$M$ & -14 & $-34,67$ & $-150,1$ & 224,8 & 247,5 & 341,5 & 247,5 & 224,8 & $-150,1$ & $-34,67$ & -14 \\
\hline
\end{tabular}

\section{Conclusion}

Existing calculation methods consider and calculate cross foundations as structures lying on a linearly deformable foundation. However, most of them do not take into account the effect of torques on their work.

\section{References}

[1] SIMVULIDI, I. KIKAVA, T. BULATOV, V. 1986. Approximate method for analysis of a beam on elastic base. Moscow., VINITI 2341-86. pp. 1-7.

[2] SIMVULIDI, I. 1987. Analysis of the engineering constructions on elastic base. Moscow, Higher School.

[3] B. Saint-Venant "Memoire sur les lignes courbes non planes, presente al' Academie des sciences le 16 September 1844. (Paris, Bachelier, 1845).

[4] KIKAVA, T. 2012. Calculation of the cross beams lying on the soil basis. Belgorod (Russian). Scientific jurnal №14(126) pp. 65-67. E-mail: belgorod@rusnaika.com.

[5] OVECHKIN, A. 1936. Calculation the beams lying on elastic base. Moscow., Aviation Institute.
[6] KIKAVA, T. 2012. The mechanic of soils and foundations. Batumi., Textbook. Batumi State University (Georgia).

[7] SIMVULIDI, I. KIKAVA, T. BULATOV, V. 1986. Relocation of elastic half-space under effect of load equally distributed along load line. Moscow., VINITI 2342-B86. pp. 2-11.

[8] LAMBE. I, WHITMAN. R. Soli mechanics. New York, 2010.

[9] HARR. M, Fundamentals of Soil Mechanics. Translation from English. Moscow; 1971. Russia.

[10] DALMATOV B. Soil Mechanics, Basements and Foundations. Moscow; 1981. Russia.

[11] TSCHEBOTARIOFF. G. Foundations. New York., 1973.

[12] KIKAVA. T, the analysis of the rectangular closed rigid frame supported by trestle bents. Tbilisi 2007. Gournal "Energy" №1. pp. 109-111. Georgia.

[13] ATKINSON. G, The mechanics of soils and foundations. Taylor and Francis Group. London and New York, 2010.

[14] KIKAVA. T, The analysis of the tunnel with the middle soilbased wall. Technical university of Ostrava. Check Republic,2016. Vol.16, №1 pp. 25-32.

[15] KIKAVA. T, Issues Related to Pile Grillage Calculation. Iris Publishers, Civil Engineering. San Francisco, CA, USA, 2019. 